\title{
Échafaudages protéiques et signalisation modulaire via les récepteurs à sept domaines transmembranaires : au-delà des protéines $\mathbf{G}$
}

Jusqu'à récemment, la cascade d'activation des récepteurs couplés aux protéines $\mathrm{G}$ (RCPG) semblait être linéaire et n'impliquer que les protéines $G$ hétérotrimériques. De nouveaux partenaires ainsi que de nouvelles voies de signalisation sont aujourd'hui identifiés. Ainsi, les kinases spécifiques aux RCPC et les arrestines, impliquées dans leur découplage des protéines $\mathrm{G}$, semblent aussi activer d'autres voies de signalisations en servant de protéines adaptatrices ou effectrices. L'interaction directe entre les RCPG et des petites protéines $G$ (Rho, ARF), des canaux ioniques, ou encore des protéines d'échafaudages via des motifs spécifiques $(\mathrm{SH} 2, \mathrm{SH} 3)$, suggère aussi l'existence de voies de signalisation alternatives. Enfin, ces récepteurs peuvent exister sous forme homo- ou hétérodimérique et des protéines chaperons peuvent moduler l'expression et la spécificité de certains de leurs ligands.

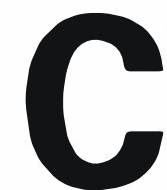

ertaines estimations émanant des projets de séquençage des génomes prédisent que les récepteurs couplés aux protéines $G$ (RCPG) forment la plus grande famille de protéines présente chez les eucaryotes. Ces récepteurs qui sont les premiers sites d'interaction pour un grand nombre de messagers chimiques tels les amines biogènes, certains acides aminés, les nucléosides et nucléotides, les hormones et neurotransmetteurs peptidiques et glycoprotéiques ainsi que les messagers lipidiquesi sont aussi impliqués dans la détection de stimuli sensoriels tels les molécules odorantes et les photons lumineux. Ainsi, ils jouent un rôle-clé dans le contrôle de processus biologiques aussi diverses que la neurotransmission, le métabolisme, la sécrétion, la différenciation et la croissance cellulaire, les réponses immunitaires et inflammatoires, l'olfaction et la vision. Cette grande diversité d'action en fait

\section{Michel Bouvier \\ Stéphane A. Laporte Monique Lagacé Marc G. Caron}

M. Bouvier, M. Lagacé: Département de biochimie et Groupe de recherche sur le système nerveux autonome, Université de Montréal, CP 6128, succursale centre-ville, Montréal, Québec, H3 C 3J7, Canada.

S.A. Laporte, M.G. Caron: Howard Hughes Medical Institute and Department of Cell biology Duke University, Durham, North Carolina, États-Unis.

des cibles pharmacologiques privilégiées pour le développement de médicaments avec des applications dans tous les champs d'activités cliniques et justifie les recherches intensives dont ils ont été l'objet au cours du dernier quart de siècle.

L'ensemble des travaux réalisés pendant cette période a permis de proposer un modèle général qui rend compte de plusieurs des propriétés fonctionnelles de cette classe de récepteurs. Selon ce modèle (figure 1), I'unité de signalisation de base se compose de trois éléments: (1) un récepteur, (2) une protéine $G$ hétérotrimérique et (3) un effecteur. La cascade de signalisation peut aussi être séparée en trois étapes: 1) Initiation du signal. La signalisation débute lorsque le messager chimique lie le récepteur et favorise son isomérisation vers une conformation active. Dans cet état activé, le récepteur peut interagir et stimuler une protéine $G$ hétéro-trimérique en catalysant 


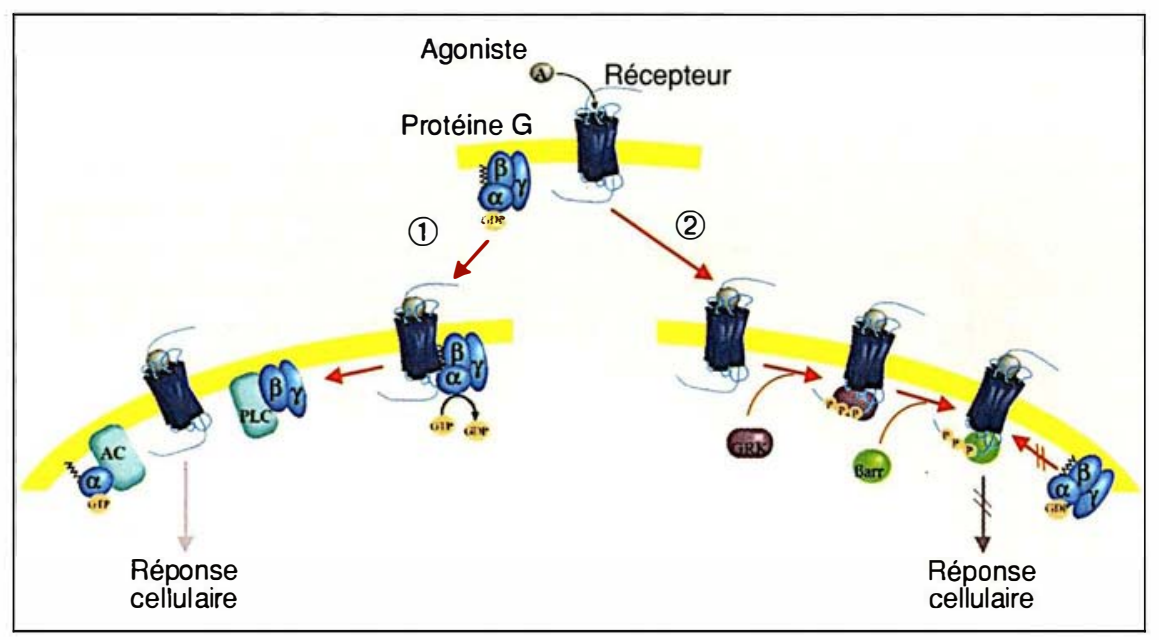

Figure 1. Représentation schématique de la voie classique de transduction du signal et de la désensibilisation des récepteurs couplés aux protéines $G$ (RCPG). La liaison d'un agoniste aux RCPG entraîne une série d'événements moléculaires qui mènent d'une part à l'activation de voies de signalisation (1) et d'autre part à la désensibilisation de la réponse (2). (1) Les récepteurs activés se lient à une protéine $G$ hétérotrimérique et catalysent l'échange d'une molécule de GDP pour une molécule de GTP sur la sous-unité $\alpha$. Ceci provoque la dissociation de la sous-unité $\alpha$ du dimère $\beta \gamma$ qui en retour interagissent avec des effecteurs (c'est-àdire adénylyl cyclase ou phospholipase C) pour réguler la génération de seconds messagers et provoquer à une réponse cellulaire appropriée. (2) En revanche, l'activation prolongée des RCPG provoque une diminution de la réponse cellulaire. Ce phénomène de désensibilisation résulte principalement de la phosphorylation des récepteurs par des kinases spécifiques des RCPG (GRK, G protein receptor kinase). Une fois phosphorylés, les récepteurs lient la protéine régulatrice $\beta$-arrestine ( $\beta$-arr) ce qui empêche le couplage subséquent des récepteurs aux protéines $G$ et donc atténue la réponse cellulaire correspondante.

l'échange d'un GDP pour un GTP à un site spécifique de liaison des nucléotides guanylés sur la sous-unité $\mathrm{G} \alpha$. Cette étape implique la formation d'un complexe ternaire formé du ligand, du récepteur et de la protéine $\mathrm{G}$ au cœur duquel, des interactions allostériques initient la transduction du signal. 2) Propagation du signal. Une fois activée, la protéine $\mathrm{G}$ se dissocie en une sous-unité $\alpha$ liée au GTP et un dimère $\beta \gamma$. Ces deux entités peuvent par la suite interagir avec différents effecteurs (enzymes, canaux, transporteurs) pour moduler leur activité et contrôler les niveaux intracellulaires de seconds messagers, $d^{\prime}$ ions et de nutriments qui influenceront l'activité cellulaire. Dans ce modèle, la sélectivité de signalisation est déterminée par la nature de la (ou des) protéine $G$ qui est recrutée par le récepteur et des effecteurs dont l'activité peut être modulée par cette protéine G. 3) Arrêt du signal. Le signal plexité des réponses cellulaires, ces modèles posent la question de la sélectivité de signalisation. En effet, comment la spécificité des réponses évoquées par des stimuli distincts estelle préservée à travers de tels réseaux qui sont en apparence largement interconnectés ?

Plusieurs études récentes permettent d'entrevoir des ébauches de réponses à cette question importante. En particulier, la mise en évidence de complexes protéiques dont l'organisation modulaire peut être réglée dans le temps, selon le niveau et le type d'activation, et dans l'espace, par leur localisation dans des compartiments spécialisés de la membrane plasmique, offre une piste intéressante [3]. L'identité des protéines présentes dans le complexe et leur site d'assemblage détermineraient le type de réponse obtenu dans des conditions particulières assurant une sélectivité d'action alors que la capacité de former plusieurs types de complexes dans des conditions distinctes assureraient la diversité nécessaire à l'élaboration de réponses biologiques complexes (figure 2). La reconnaissance que certains de ces modules protéiques pourraient ne pas nécessiter la présence de protéines $G$ hétéro-trimériques pour leurs fonctions de signalisation laisse entrevoir l'existence de modes de transduction des signaux insoupçonnés jusqu'à maintenant pour les RCPG.

Dans cette revue nous discuterons de plusieurs études récentes qui illustrent l'importance des interactions protéine-protéine et de la formation de complexes protéiques dans la signalisation par les RCPG. Ceci entraîne la réassociation de I'hétéro-trimère $\alpha \beta \gamma$ inactif portant une molécule de GDP ce qui ramène le système à son état basal. Les détails de ce modèle de fonctionnement des RCPG ont été revus récemment [1].

A ce mode de signalisation en cascades linéaires se superpose un haut niveau d'interconnexion qui permet d'expliquer des phénomènes de divergences et de convergences des signaux. En particulier, les interactions entre les voies des RCPG et celles des récepteurs à activité tyrosine kinases des facteurs de croissance ont mené au concept de réseaux interconnectés qui, à l'image d'une toile d'araignée, permettent une intégration en de multiples points des signaux perçus par la cellule [2]. Bien que rendant compte de la com-

\section{Rôles des protéines régulatrices et des microdomaines de la membrane plasmique}

Les réponses cellulaires résultantes de I'activation de RCPG sont contrôlées par l'étroite balance qui existe entre I'initiation et la terminaison de cette activation. En plus du mécanisme décrit plus haut et qui permet l'arrêt de la signalisation en désactivant les protéines $G$, un processus prenant place à plus long terme permet de contrôler le niveau de réactivité cellulaire en agissant directement au niveau des récepteurs. Ce phéno- 


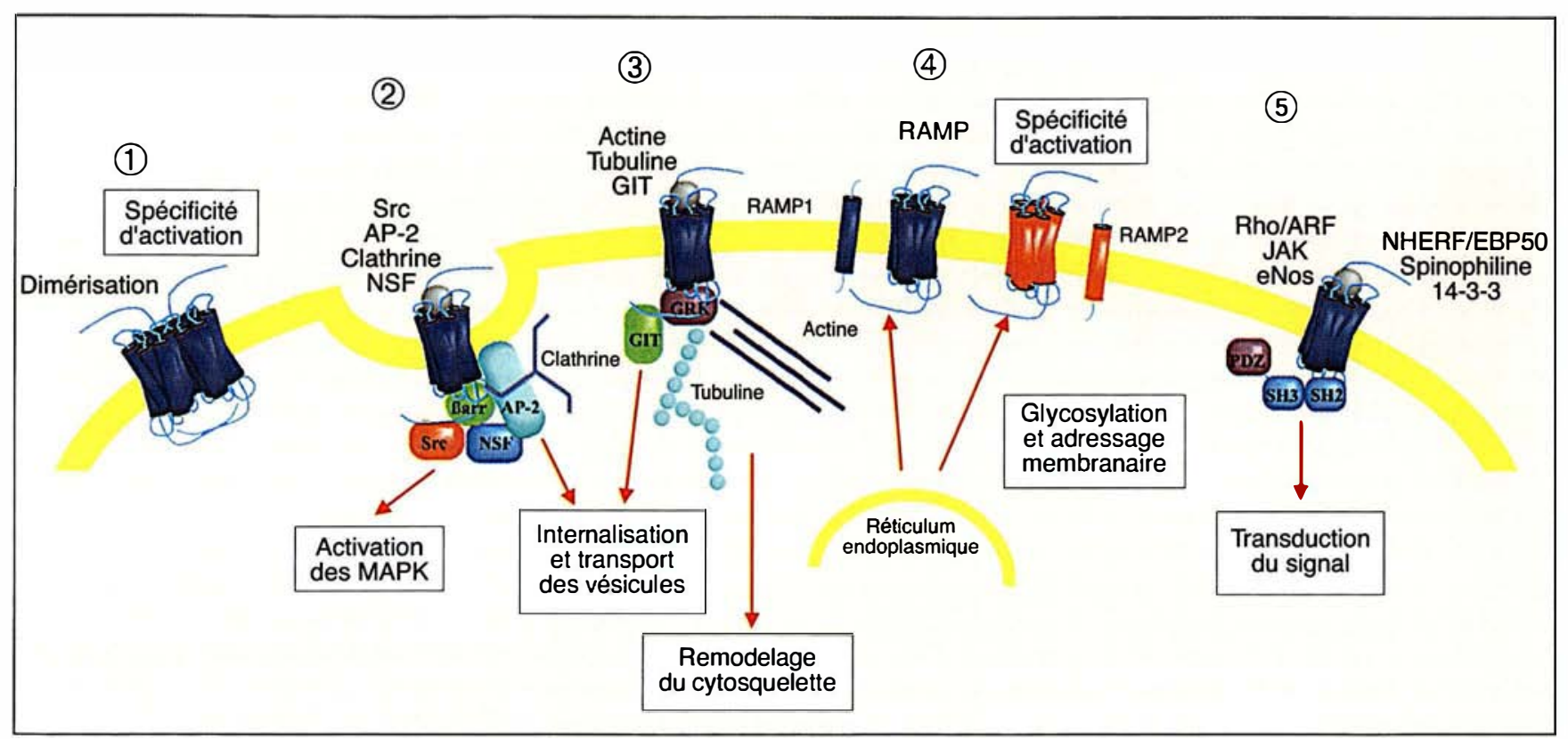

Figure 2. Échafaudages protéiques et signalisation modulaire. Outre le couplage des récepteurs aux protéines $G$, les RCPG peuvent former plusieurs types de complexes protéiques qui résultent en une diversification des signaux intracellulaires. (1) La formation de complexes dimériques entre certains RCPG pourrait contribuer aux processus d'activation et à la spécificité d'action de ceux-ci. (2) L'association des RCPG à des protéines adaptatrices qui sont impliquées dans la désensibilisation des récepteurs, tels que la $\beta$-arrestine, participerait à l'activation de la voie des MAP-kinases en permettant le recrutement de la forme active de la kinase Src et l'adressage du complexe vers les puits tapissés de clathrine. De plus, l'interaction de la $\beta$-arrestine avec la clathrine et les protéines adaptatrice AP-2 (adaptine) et NSF (Nethylmaleimide sensitive factor) jouerait un rôle actif dans l'endocytose et le transport des récepteurs vers différents compartiments intracellulaires. (3) L'identification de substrats pour les GRK comme l'actine et de la tubuline et l'association d'un facteur activateur de I'hydrolyse du GTP spécifique aux petites protéines $G$ de type ARF (GIT), suggère un rôle important de ces kinases dans le remodelage du cytosquelette et dans le transport vésiculaire. (4) L'association de certains RCPG avec des protéines escortes (RAMPS, receptor-activity-modifying proteins) assure la maturation et l'adressage adéquat des récepteurs à leur sortie du réticulum endoplasmique. L'association stable de ces récepteurs avec des isoformes particulières de RAMP détermine aussi leurs propriétés pharmacologiques. (5) L'existence potentiel d'interactions directes entre les RCPG et des petites protéines $G$ de type Rho/ARF, des kinases de la famille des Januskinases (JAK), des enzymes (eNOS) ou encore des protéines d'échafaudage telles que NHERF/EBP50 ( $\mathrm{Na}^{+} / \mathrm{H}^{+}$exchanger regulatory factor/ezrin-radixin-moesin binding phosphoprotein-50), spinophiline et 14-3-3, suggère l'existence de voies de signalisation alternatives qui seraient indépendantes des protéines $G$ hétéro-trimériques.

mène biologique connu sous le nom de désensibilisation, implique la phosphorylation des récepteurs par des protéine-kinases spécifiques des RCPG (GRK) et la liaison subséquente des protéines régulatrices arrestines (figure 1). Les arrestines inhibent de façon compétitive le couplage des récepteurs à leurs protéines $G$ et entraînent l'internalisation des récepteurs en interagissant directement avec la clathrine des puits d'endocytose (pour une revue, voir [4]).

En plus de leur rôle classique dans la désensibilisation des voies de signalisation liées aux protéines $G$, les arrestines semblent participer à la formation de complexes protéiques menant à l'activation de voies de signalisation alternatives. En effet, il a récemment été démontré que la stimulation du récepteur $\beta_{2}$-adrénergique entraîne la formation d'un complexe comprenant le récepteur, la $\beta$-arrestine-1 et c-Src activé [5]. Ainsi, la $\beta$-arrestine-1 semble jouer un rôle de protéine adaptatrice dans le recrutement et l'activation de tyrosine kinases non réceptorielles grâce à une interaction entre le domaine $\mathrm{SH} 3$ de $\mathrm{Src}$ et une région riche en proline de la $\beta$-arrestine-1. L'importance fonctionnelle de cette interaction est illustrée par I'observation que des formes mutantes de la $\beta$-arrestine-1 qui ne peuvent plus s'associer à Src mais peuvent toujours lier le récepteur et la clathrine ont des effets dominants négatifs sur l'activation des MAPkinases Erk-1 et Erk-2 induite par I'activation des récepteurs $\beta_{2}$-adrénergiques. Par ailleurs, des mutants de la $\beta$-arrestine-1 qui ne peuvent interagir avec la clathrine mais s'associent normalement au récepteur et à Src inhibent aussi cette voie de signalisation suggérant que non seulement le recrutement de Src mais aussi le ciblage du complexe vers les puits tapissés de clathrine est nécessaire à l'activation des MAP-kinases. Ainsi, l'échafaudage protéique aurait pour fonction le recrutement à la membrane de la forme active de la kinase Src, le ciblage du complexe récepteur- $\beta$-arrestine-Src vers les puits de clathrine et l'activation de la cascade 
de signalisation conduisant à la stimulation de Ras et ultimement à l'activation des MAP-kinases.

Un des aspects fascinants de ces observations réside dans le fait que le recrutement de Src et le ciblage du complexe vers les puits de clathrine résultent $d^{\prime}$ un processus qui conduit au découplage fonctionnel et physique du récepteur de sa protéine $G$ (Gs dans le cas du récepteur $\beta_{2}$-adrénergique) et à la désensibilisation de la réponse de son effecteur classique (I'adênylyl cyclase dans ce cas). Ainsi, il semblerait que c'est un récepteur "désensibilisé " qui peut activer la voie des MAP-kinases. Ceci nous force donc à reconsidérer les événements de désensibilisation résultants de la phosphorylation des récepteurs par les GRK et de la liaison de la $\beta$-arrestine non plus comme une inactivation du récepteur mais plutôt comme une réorganisation modulaire différente de partenaires protéiques favorisant une voie de signalisation au détriment $d^{\prime}$ une autre.

L'identification récente de l'actine et de la tubuline comme substrats potentiels des GRK [6-9] permet de proposer que ces kinases puissent jouer un rôle de signalisation qui pourrait même dépasser ceux évoqués plus haut. Elles pourraient entre autre être impliquées dans le remodelage du cytosquelette qui accompagnent la stimulation de certains RCPG. L'association de GRK-2 à un facteur activateur de l'hydrolyse du GTP spécifique aux petites protéines $G$ de type ARF (ARF-GAP), nommé GIT1 [10], conforte l'idée d'un rôle plus large pour ces kinases.

Nous avons évoqué plus haut le rôle que joue les arrestines dans l'endocytose des récepteurs en s'associant à la clathrines [11] et à la protéine adaptatrice AP-2 [12]. Un criblage par la méthode de double hybrides en levure, a récemment révélé que la $\beta$ arrestine-1 peut aussi s'associer à une protéine impliquée dans les processus de fusion membranaire, la "N-ethylmaleimide sensitive factor" (NSF) [13]. Bien que le rôle précis de cette interaction reste à être déterminé, il est tentant de spéculer qu'elle pourrait être responsable de la fusion des endosomes et/ou du ciblage des complexes de signalisation vers des sousdomaines précis de la membrane plasmique. Le rôle que GIT1 et ARF pourraient jouer dans ces processus suite à leur recrutement par GRK-2 devra aussi faire l'objet d'études plus détaillées.

Les puits tapissés de clathrine ne sont pas les seuls domaines spécialisés de la membrane plasmique ayant été impliqués dans la transduction des signaux par les RCPG. En effet, plusieurs études récentes suggèrent que certains RCPG soient localisés préférentiellement dans des domaines riches en lysosphyngolipides et cholestérol contenant ou non la protéine cavéoline. Ces domaines, connus sous le nom de "raft" (radeau) lorsque dépourvu de cavéoline ou cavéolae (petite cave) lorsqu'ils en contiennent, seraient des régions privilégiées permettant I'assemblage de plusieurs protéines impliquées dans la signalisation cellulaire [14]. Bien que spéculative, l'idée que les "raft/cavéolae" et les puits tapissés de clathrine puissent favoriser la formation de modules distincts de signalisation permet d'envisager un confinement spatial des signaux qui pourrait contribuer à une forme de sélectivité d'action.

\section{Signalisation indépendante des protéines $\mathbf{G}$ hétéro-trimériques}

Jusqu'à tout récemment, il était généralement admis que tous les événements de signalisation en aval d'un RCPG résultaient directement ou indirectement de l'activation d'une protéine $G$ hétéro-trimérique. Toutefois, plusieurs études récentes ont remis ce dogme en question en proposant l'existence de voies de signalisation alternatives qui seraient indépendantes des protéines $G$ hétérotrimériques.

Parmi ces voies non classiques, l'existence d'un complexe protéique entre le récepteur muscarinique $M 3$ d'une part et une petite protéine $G$ de type Rho/ARF d'autre part [14] a particulièrement attiré l'attention. Ce complexe, dont la formation dépend de l'activation du récepteur, a été impliqué dans la stimulation de la phospholipase $\mathrm{D}$ dans des cellules astrocytaires humaines. Dans ces cellules, I'activation de la phosphilipase D par l'acétylcholine est bloquée par des inhibiteurs pharmacologiques et biochimiques agissant sur les protéines ARF et Rho mais non par des agents affectant les protéines $G$ hétéro-trimériques ou la phospholipase C. Un motif NPXXY localisé à l'interface cytosolique du septième domaine transmembranaire du récepteur semblant jouer un rôle crucial dans le recrutement de ARF/Rho [15], il est permis de croire qu'il puisse s'agir $d^{\prime}$ une voie de signalisation générale pour les RCPG. En effet, ce motif est conservé à une position similaire dans un grand nombre de récepteurs de cette famille.

D'autres exemples d'activation par voies non conventionnelles incluent le recrutement et I'activation de membres de la famille des Janus kinases (JAK) par les récepteurs CCR2B des chémokines [16] et AT1 de I'angiotensine II [17]. JAK est une famille de tyrosine kinases dont le rôle a d'abord été apprécié dans la signalisation émanant des récepteurs des interférons $\alpha$ et $\gamma$. Classiquement, ces kinases sont impliquées dans I'activation transcriptionnelle de plusieurs gènes en phosphorylant des facteurs transcriptionnels connus sous le nom de STAT. Ainsi, le recrutement de JAK par certains RCPG suggère I'existence dune voie de régulation de l'expression génique par ces derniers qui n'impliquerait aucune protéine $G$.

Une autre interaction directe entre un RCPG et une molécule effectrice a récemment été démontrée pour le récepteur de la bradykinine et I'enzyme NO synthase endothéliale (eNOS) [18]. Dans ce cas, la liaison de la bradykinine à son récepteur entraîne la dissociation du complexe relevant ainsi l'inhibition de l'activité de la eNOS qui résultait de son association avec le récepteur. Le domaine du récepteur de la bradykinine responsable de cette action inhibitrice réside dans une séquence de 24 acides aminés situés dans la portion carboxyl-terminal du récepteur [19] qui à elle seule peut inhiber I'activité de l'enzyme. La généralisation possible de l'interaction entre la eNOS et un RPCG a été démontrée par la liaison de eNOS à un peptide dérivé du domaine carboxyl-terminal du récepteur AT1 de I'angiotensine II in vitro [18].

Finalement, une étude récente a démontré l'existence d'une interac- 
tion directe entre la queue cytoplasmique du récepteur dopaminergique D5 et la seconde boucle intracellulaire de la sous-unité $\gamma 2$ du récepteur ionotropique $\operatorname{GABA}(A)$ [20]. L'implication fonctionnelle de cette interaction est suggérée par l'inhibition de l'activité signalétique qui est observé pour les deux récepteurs lorsque ceux-ci sont co-exprimés. La présence de récepteur dopaminergique D5 dans certaines projections neuronales GABAergiques [21] offre une base anatomique à ce mécanisme de régulation réciproque direct. La possibilité que ce type $d^{\prime}$ 'interaction entre les récepteurs dopaminergiques et GABA puisse être généralisé à d'autres RCPG et canaux ioniques laisse entrevoir de nouvelles avenues de recherche.

\section{Motifs et protéines d'échafaudages}

L'identification d'un nombre grandissant de protéines pouvant s'associer aux RCPG a poussé plusieurs chercheurs à utiliser des séquences protéiques spécifiquement retrouvées dans des domaines intracellulaires de récepteurs comme appâts moléculaires. En plus de permettre l'identification de molécules effectrices telles que celle citées plus haut, cette approche a permis d'isoler plusieurs partenaires $n^{\prime}$ ayant pas d'activité enzymatique propre leur permettant d'agir comme effecteur. Toutefois, plusieurs de ces protéines contiennent des motifs $d$ 'interaction protéine-protéine, qui leur permettraient de servir de molécules d'échafaudages impliquées dans la modulation de certains effecteurs et/ou dans l'assemblage de complexes multi-protéiques de signalisation.

Une de ces protéines, connue sous le nom de $\mathrm{Na}^{+} / \mathrm{H}^{+}$Exchanger Regulatory Factor (NHERF) ou Ezrin-RadixinMoesin Binding Phosphoprotein-50 (EBP50), a récemment été identifiée pour sa capacité à lier la séquence canonique $S / T \times L$ situé à l'extrémité $C$ terminale du récepteur $\beta_{2}$-adrénergique [22]. Cette interaction qui a lieu via un des deux domaines PDZ (ainsi appelé pour leur homologie avec les protéines PSD-95, Discslarge et ZO-1) de NHERF/EBP50, et qui est favorisée par l'activation du récepteur, semble jouer deux rôles fonctionnels distincts. D'une part, comme l'un de ses deux noms I'indique, NHERF/EBP50 interagit avec le co-transporteur $\mathrm{Na}^{+} / \mathrm{H}^{+}$ (Nhe3) et ce faisant inhibe son activité. Le recrutement de NHERF/ EBP50 par le récepteur $\beta_{2}$-adrénergique conduit ainsi à une désinhibition du transporteur en le libérant de ce régulateur négatif [23]. D'autre part, une fois associé au récepteur, NHERF/EBP50 joue un rôle dans le processus d'endocytose grâce à son interaction simultanée avec la protéine ezrine permettant un lien entre le récepteur et des éléments du cytosquelette [24]. L'association du récepteur $\beta_{2}$-adrénergique et de NHERF/EBP50 favorise donc le désassemblage d'un complexe qui maintenait Nhe3 inactif au profit d'un complexe récepteur-NHERF/EBP50-ezrine qui régule le transport intracellulaire du récepteur.

Une association entre un RCPG et des éléments du cytosquelette a aussi été suggérée par l'identification de la spinophiline comme partenaire direct du récepteur dopaminergique D2 dans une étude de criblage par double hybride en levure [25]. En effet, en plus de pouvoir lier le récepteur, cette protéine peut interagir avec les phosphatases de type 1 et l'actine. Il a donc été proposé que la spinophiline puisse permettre l'établissement d'un complexe de signalisation liant le récepteur D2, une phosphatase et le cytosquelette d'actine. Le fait qu'aucune de ces interactions n'impliquent I'unique domaine PDZ de la spinophiline suggère qu'une autre protéine puisse participer au complexe. Toutefois, ni le rôle physiologique ni même l'existence d'un tel complexe n'a été démontrée à ce jour.

Une étude de liaison en milieu solide, en utilisant la troisième boucle intracellulaire du récepteur $\alpha_{2}$-adrénergique comme sonde, a mené à l'isolation $d$ 'une autre protéine d'échafaudage, le sous-type $\zeta$ de la protéine 14-3-3 [26]. Cette protéine qui s'exprime de façon ubiquitaire, peut interagir avec diverses protéines impliquées dans la transduction des signaux tels que la kinase Raf, la phosphatidylinositol-3-kinase et des phosphatases pour moduler leur activité. Toutefois, comme dans le cas de la spinophiline, l'importance fonc- tionnelle de l'association de 14-3-3 $\zeta$ au récepteur $\alpha_{2}$-adrénergique reste encore spéculative.

Finalement, quelques études ont pu mettre en évidence des interactions entres des protéines contenant des domaines $\mathrm{SH} 3$ [27] ou $\mathrm{SH} 2$ [28] et des boucles cytosoliques de divers RCPG. De nouveau, la formation de ces complexes pourraient être à l'origine de modes alternatifs de signalisation pour les RCPG mais leur importance physiologique de même que les conditions particulières dans lesquelles ils pourraient jouer un rôle reste à démontrer.

\section{Protéines escortes}

Récemment, il a été montré que certains RCPG incluant les opsines, $[29,30]$ les récepteurs olfactifs [31] et le récepteur du CGRP (calcitoningene-related peptide) [32] requièrent la présence de protéines escortes/ chaperonnes spécialisées pour leur expression normale à la surface des cellules. Bien que certaines de ces protéines semblent surtout importantes pour assurer le repliement adéquat des récepteurs, d'autres restent associées aux récepteurs même lorsque ceux-ci atteignent la surface cellulaire et, affectent leurs fonctions.

Le cas le mieux caractérisé d'une telle association stable d'une protéine escorte avec un RCPG concerne le récepteur du CGRP. Des travaux récents ont en effet permis de déterminer que l'association d'une protéine possédant un seul domaine transmembranaire est indispensable à I'expression du récepteur du CGRP. Cette découverte fut réalisée au terme de multiples efforts pour identifier le gène codant pour ce récepteur quand McLatchie et al. [32] réussirent à isoler un nouveau gène qui, lorsque exprimé avec le gène du CRLR (calcitonin-receptor-like-receptor), confère une réponse de liaison et de signalisation au CGRP. La nouvelle protéine fut donc considérée comme un corécepteur et les trois isoformes clonés appelées receptor-activity-modifying proteins (RAMP 1, 2, et 3).

Bien que la co-expression du CRLR avec RAMP1 résulte dans I'expression d'un récepteur du CGRP, sa coexpression avec RAMP2 ou RAMP3 donne naissance à un récepteur qui a 
les propriétés pharmacologiques d'un récepteur de l'adrénoméduline. Ces résultats suggèrent donc que les propriétés pharmacologiques d'un RCPG puissent être déterminées par la nature des RAMP qui y sont associés. Les propriétés pharmacologiques distinctes s'accompagnent aussi de différences biochimiques. Ainsi, alors que la co-expression du CRLR avec RAMP1 résulte dans un récepteur portant des sucres complexes, la présence de RAMP 2 ou de RAMP 3 mène à un récepteur avec des chaînes d'hydrocarbures riches en manoses. Ces différences indiquent que les RAMP dictent des voies de maturations distinctes pour le CRLR nouvellement synthétisé. Associé à I'observation que le CRLR exprimé en absence de RAMP est retenu dans la cellule au niveau du réticulum endoplasmique, I'ensemble des résultats suggère que les RAMP représentent des protéines chaperonnes/escortes spécialisées qui sont requises pour la maturation, le transport et la fonction de certains RCPG. La large distribution tissulaire des RAMP suggère qu'ils puissent jouer un rôle qui ne se limite pas au récepteur du CGRP et de l'adrénomoduline.

II reste cependant à déterminer si la fonction des RAMP se situe principalement au niveau de la maturation et du ciblage des RCPG ou s'ils agissent aussi comme modulateurs directs d'activité par le biais d'interactions intermoléculaire avec les récepteurs une fois ceux-ci à la membrane plasmique. De même, bien que les études réalisées jusqu'à maintenant démontrent clairement que les RAMP ont un effet dramatique sur les propriétés pharmacologiques de certains RPCG, aucune donnée n'est disponible sur l'influence qu'ils pourraient avoir sur les voies de signalisation qui sont engagées par ces récepteurs.

\section{Dimérisation des récepteurs}

L'homo-dimérization est un type d'assemblage protéique très souvent observé et plusieurs des partenaires impliqués dans la transduction des signaux existent sous cette forme. En particulier, une grande majorité des récepteurs membranaires sont soit constitutivement dimérique ou le deviennent lorsque lié par un ago- niste. Jusqu'à tout récemment, les RCPG semblaient faire exception à cette règle, les divers modèles décrivant leur mode d'activation les présentant comme des entités monomériques. Toutefois, une série d'études récentes ont remis en question ces modèles en présentant de nombreuses évidences suggérant l'existence et la fonctionnalité de RCPG dimérique.

Malgré les évidences remontant au début des années 1980 qui suggéraient l'existence de dimères de RCPG (pour une revue, voir [33]), il a fallu attendre 1993 et une étude élégante de Maggio et al. [34] pour que l'idée des dimères de RCPG suscite l'intérêt de la communauté scientifique. Dans cette étude, la co-expression de récepteurs chimériques (muscarinique $\mathrm{M} 3 / \alpha_{2}$-adrénergique et $\alpha_{2}$ adrénergique/muscarinique $M 3$ ) permettait de conférer une activité de liaison et de signalisation pour des ligands muscariniques et adrénergiques alors que l'expression de chacune de ces chimères individuellement $n^{\prime}$ instaurait aucune activité biologique [34]. II a donc été proposé que la dimérisation entre les deux récepteurs chimériques soit responsable de la formation des deux sites de liaison et assurait la complémentarité fonctionnelle.

Sur le plan biochimique, la co-immunoprécipitation de récepteurs portant des épitopes immunologiques distincts, tout d'abord réalisé pour le récepteur $\beta 2$-adrénergique [35] puis confirmée pour plusieurs autres RCPG [36-39], représente la première évidence directe confirmant I'existence $d$ 'homo-dimères de RCPG. Pour le récepteur $\beta_{2}$-adrénergique, un motif d'interaction hydrophobe localisé dans le $6^{\mathrm{e}}$ domaine transmembranaire a été proposé comme site potentiel de dimérisation. Un peptide synthétique, mimant cette séquence, inhibe de façon importante la capacité du récepteur $\beta_{2}$-adrénergique à stimuler l'activité de l'adénylyl cyclase tout en prévenant la formation de dimer, confirmant un rôle fonctionnel pour la dimérisation [35]. Plus récemment, l'étude des récepteurs métabotropiques de l'acide $\gamma$ amino-butyrique (GABA) a grandement renforcé I'hypothèse $d^{\prime}$ 'une dimérisation fonctionnelle des RCPG. Plusieurs groupes ont en effet simulta- nément découvert que la co-expression de deux isoformes de ces récepteurs, $G_{A B A} R 1$ et $G_{B B A} R 2$, est nécessaire à la formation $d^{\prime}$ un récepteur GABA fonctionnel à la surface cellulaire [39-43]. En fait, lorsque exprimé seul, le récepteur $\mathrm{GABA}_{B} \mathrm{R} 1$ est incomplètement glycosylé et retenu dans le réticulum endoplasmique. L'isoforme $G_{A B A} R 2$, pour sa part, peut être acheminé à la membrane plasmique mais ne peut isolément lier le GABA. L'existence d'un complexe stable entre $\mathrm{GABA}_{B} \mathrm{R} 1$ et R2 a par ailleurs été confirmé par des études de co-immunoprécipitation, confirmant la nécessité de l'association physique des deux récepteurs. II a donc été proposé que $G_{A B A} R 2$ puisse servir de chaperonne/escorte à $\mathrm{GABA}_{\mathrm{B}} \mathrm{R} 1$, permettant la formation d'un complexe actif à la surface cellulaire. Le patron d'expression des deux récepteurs, se chevauchant dans plusieurs régions du cerveau qui affichent un récepteur GABA fonctionnel, ajoute à la pertinence physiologique de I'hypothèse proposée. La contribution de chacun des monomères à la liaison du GABA et à la transduction du signal reste toutefois à être déterminée.

$L^{\prime}$ interaction entre $G A B A_{B} R 1$ et $R 2$ n'est pas le seul exemple d'hétérodimérisation de RCPG. Jordan et Devi ont en effet récemment rapporté l'existence d'un complexe hétérodimérique formé de récepteurs opiacés de types $\delta$ et $\kappa$ qui possède des propriétés fonctionnelles et de liaisons distinctes de celles observées pour chacun des récepteurs exprimés individuellement. Ainsi, I'hétérodimérisation pourrait représenter un phénomène général contribuant à la diversité pharmacologique des RCPG [44].

Bien qu'assez convaincantes, les évidences biochimiques directes impliquant l'existence de dimères de RCPG étaient jusqu'à tout récemment fondées sur des études requérant la lyse cellulaire et la solubilisation des récepteurs pouvant mener à des phénomènes $d$ 'agrégation non spécifique. Toutefois, une approche biophysique tirant avantage du phénomène de transfert d'énergie bioluminescente de résonance (bioluminescence resonance energy transfer; BRET) a récemment permis de démontrer que le récepteur $\beta_{2}$-adré- 
nergique existe sous forme de dimères dans des cellules vivantes [45]. Le BRET est un transfert non radiatif d'énergie entre un donneur bioluminescent (la luciférase) et un accepteur fluorescent (la protéine fluorescente verte GFP). Lorsque le donneur et l'accepteur sont présents à une distance de $50 \AA$ ou moins l'un de l'autre, l'énergie de luminescence générée par la dégradation enzymatique du substrat de la luciférase, la co-elanterazine, est transférée à la GFP qui en retour émet de la fluorescence avec des propriétés spectrales qui lui sont propres. Dans des cellules co-exprimant des récepteurs $\beta_{2}$ adrénergique fusionnés à la luciférase et à une GFP $\left(\beta_{2} A R-R l u c\right.$ et $\beta_{2} A R$ GFP), Angers et al. ont démontré que l'addition de co-elanterazine entraîne une émission de fluorescence de la GFP révélant que le récepteur existe de façon constitutive sous forme de dimères. De plus, I'augmentation de fluorescence observée en réponse à l'addition $d$ 'un agoniste suggère que la stimulation du récepteur favorise la formation de dimères supportant un rôle important pour la dimérisation dans la transduction du signal.

\section{Conclusion}

Les évidences présentées dans cette revue illustrent bien la diversité grandissante des voies de signalisations qu'empruntent les RCPG. La découverte de voies de signalisation non conventionnelles pour les RCPG a connu un essor considérable et rapide grâce au développement de nouvelles méthodes d'études des interactions protéine-protéine qui reposent largement sur des approches réalisées in vitro. Même si le rôle physiologique de la plupart de ces interactions n'a pas encore été évalué de façon satisfaisante, il est certain que le concept de complexe protéique modulaire, qui a déjà été établi pour d'autres voies de signalisation, influencera, dans les années à venir, notre façon de voir les RCPG. En effet, la reconnaissance de l'existence de systèmes de signalisations en échafaudages pourra nous permettre de mieux comprendre les différents niveaux de spécificité spatiotemporelle requis au contrôle de réponses physiologiques complexes induites par un récepteur. Pour ce faire, il faudra toutefois que l'importance relative et la réalité physiologique des diverses interactions protéine-protéine proposées soient scrutées systématiquement. Le rôle de la dimérisation des RCPG dans la mise en place des divers modules de signalisation devra aussi être l'objet d'une attention particulière. L'utilisation de nouvelles approches biophysique, tel le BRET, qui permettent I'étude des interactions protéiques en cellules vivantes et en temps réel, sera certainement des plus utiles dans la poursuite de ces objectifs. Le nombre de protéines et de complexes qui ont récemment été impliqués dans la signalisation par les RCPG est telle que les années à venir devraient être des plus excitantes si elles doivent nous permettre de compléter cette nouvelle cartographie modulaire

\section{RÉFÉRENCES}

1. Bockaert J, Pin JP. Molecular tinkering of $G$ protein-coupled receptors: an evolutionary success. Embo J 1999; 18: 1723-9.

2. Bourne HR. Signal transduction. Team blue sees red. Nature 1995; 376: 727-9.

3. Zuker CS, Ranganathan R. The path to specificity. Science 1999; 283: 650-1.

4. Bouvier M, Nantel F, Valiquette $M$, Moffett $S$, Mouillac $B$. Le récepteur $\beta 2$-adrénergique; un modèle d'étude des mécanismes moléculaires de la désensibilisation. Med Sci 1995; 11 : 819-27.

5. Luttrell LM, Ferguson SS, Daaka $Y$, et al. Beta-arrestin-dependent formation of beta2 adrenergic receptor-Src protein kinase complexes. Science 1999; 283: 655-61.

6. Carman CV, Som T, Kim CM, Benovic JL. Binding and phosphorylation of tubulin by $G$ protein-coupled receptor kinases. I Biol Chem 1998; 273: 20308-16.

7. Freeman JL, De La Cruz EM, Pollard TD, Lefkowitz RJ, Pitcher JA. Regulation of $G$ protein-coupled receptor kinase 5 (GRK5) by actin. J Biol Chem 1998; 273 : 20653-7.

8. Pitcher JA, Hall RA, Daaka Y, et al. The G protein-coupled receptor kinase 2 is a microtubule-associated protein kinase that phosphorylates tubulin. J Biol Chem 1998; 273:12316-24.

9. Haga $K$, Ogawa $H$, Haga $T$, Murofushi $H$. GTP-binding-protein-coupled recepto kinase 2 (GRK2) binds and phosphorylates tubulin. Eur J Biochem 1998; 255: 363-8.

10. Premont RT, Claing $A$, Vitale $N$, et al. beta2-Adrenergic receptor regulation by GIT1, a G protein-coupled receptor kinaseassociated ADP ribosylation factor GTPase- activating protein. PrCc Natl Acad Sci USA 1998; 95: 14082-7.

11. Goodman OB Jr, Krupnick JG, Santini F, et al. Beta-arrestin acts as a clathrin adaptor in endocytosis of the beta2-adrenergic receptor. Nature 1996; 383: 447-50.

12. Laporte SA, Oakley RH, Zhang J, et al. The beta2-adrenergic receptor/beta-arrestin complex recruits the clathrin adaptor AP-2 during endocytosis. Proc Natl Acad Sci USA 1999; $96: 3712-7$.

13. McDonald PH, Cote NL, Lin FT, Premont RT, Pitcher JA', Lefkowitz RJ. Identification of NSF as a beta-arrestin1-binding protein. Implications for beta2-adrenergic receptor regulation. J Biol Chem 1999; 274 : 10677-80.

14. Okamoto T, Schlegel A, Scherer PE, Lisanti MP. Caveolins, a family of scaffolding proteins for organizing "preassembled signaling complexes $m$ at the plasma membrane. JBiol Chem 1998; 273: 5419-22.

15. Mitchell R, McCulloch D, Lutz E, et al. Rhodopsin-family receptors associate with small G proteins to activate phospholipase D. Nature $1998 ; 392: 411-4$.

16. Mellado M, Rodriguez-Frade JM, Aragay $A$, et al. The chemokine monocyte chemotactic protein 1 triggers Janus kinase 2 activation and tyrosine phosphorylation of the CCR2B receptor. J Immunol 1998; 161: 805-13.

17. Ali MS, Sayeski PP, Dirksen LB, Hayzer DJ, Marrero MB, Bernstein KE. Dependence on the motif YIPP for the physical association of Jak2 kinase with the intracellular carboxyl tail of the angiotensin II AT1 receptor. I Biol Chem 1997; 272: 23382-8.

18. Ju $\mathrm{H}$, Venema VJ, Marrero MB, Venema RC. Inhibitory interactions of the bradykinin B2 receptor with endothelial nitric-oxide synthase. J Biol Chem 1998; 273: 24025-9.

19. Golser $\mathrm{R}$, Gorren AC, Leber $\mathrm{A}$, et al. Interaction of endothelial and neuronal nitric-oxide synthases with the bradykinin B2 receptor. Binding of an inhibitory peptide to the oxygenase domain blocks uncoupled NADPH oxidation. I Biol Chem 2000; 275: 5291-6.

20. Liu F, Wan Q, Pristupa ZB, Yu XM, Wang YT, Niznik HB. Direct protein-protein coupling enables cross-talk between dopamine D5 and gamma-aminobutyric acid A receptors. Nature $2000 ; 403$ : 274-80.

21. Bergson C, Mrzljak L, Lidow MS, Goldman-Rakic PS, Levenson R. Characterization of subtype-specific antibodies to the human D5 dopamine receptor: studies in primate brain and transfected mammalian cells. Proc Natl Acad Sci USA 1995; 92: 3468-72.

22. Hall RA, Ostedgaard LS, Premont RT, et al. A C-terminal motif found in the beta2adrenergic receptor, $\mathrm{P} 2 \mathrm{Y} 1$ receptor and cystic fibrosis transmembrane conductance regulator determines binding to the $\mathrm{Na}^{+} / \mathrm{H}^{+}$ exchanger regulatory factor family of PDZ proteins Proc Natl Acad Sci USA 1998. 95 . 8496-501. 


\section{RÉFÉRENCES}

23. Hall RA, Premont RT, Chow CW, et al. The beta2-adrenergic receptor interacts with the $\mathrm{Na}^{+} / \mathrm{H}^{+}$-exchanger regulatory factor to control $\mathrm{Na}^{+} / \mathrm{H}^{+}$exchange. Nature 1998; $392: 626-30$.

24. Cao TT, Deacon HW, Reczek D, Bretscher A, von Zastrow M. A kinase-regulated PDZ-domain interaction controls endocytic sorting of the beta2-adrenergic receptor. Nature 1999; 401: 286-90.

25. Smith FD, Oxford GS, Milgram SL. Association of the D2 dopamine receptor third cytoplasmic loop with spinophilin, a protein phosphatase-1-interacting protein. J Biol Chem 1999; 274: 19894-900.

26. Prezeau L, Richman JG, Edwards SW, Limbird LE. The zeta isoform of 14-3-3 proteins interacts with the third intracellular loop of different alpha2-adrenergic receptor subtypes. J Biol Chem 1999; 274: 13462-9.

27. Tang Y, Hu LA, Miller WE, et al. Identification of the endophilins (SH3p4/p8/p13) as novel binding partners for the beta1-adrenergic receptor. Proc Natl Acad Sci USA 1999; 96: 12559-64.

28. Karoor V, Wang L, Wang HY, Malbon CC. Insulin stimulates sequestration of betaadrenergic receptors and enhanced association of beta-adrenergic receptors with Grb2 via tyrosine 350. J Biol Chem 1998; 273: 33035-41.

29. Baker EK, Colley NJ, Zuker CS. The cyclophilin homolog NinaA functions as a chaperone, forming a stable complex in vivo with its protein target rhodopsin. EMBO J 1994; 13: 4886-95.

30. Ferreira PA, Nakayama TA, Pak WL, Travis $\mathrm{GH}$. Cyclophilin-related protein RanBP2 acts as chaperone for red/green opsin. Nature 1996; 383:637-40.

31. Dwyer ND, Troemel ER, Sengupta P, Bargmann $\mathrm{Cl}$. Odorant receptor localization to olfactory cilia is mediated by ODR-4, a novel membrane-associated protein. Cell 1998; 93: 455-66.

32. McLatchie LM, Fraser NJ, Main MJ, et al. RAMPs regulate the transport and ligand specificity of the calcitonin-receptor-like receptor. Nature 1998; 393: 333-9.

33. Hebert TE, Bouvier M. Structural and functional aspects of $G$ protein-coupled receptor oligomerization. Biochem Cell Biol 1998; 76: $1-11$.

34. Maggio R, Vogel Z, Wess J. Coexpression studies with mutant muscarinic/adrenergic receptors provide evidence for intermolecular "cross-talk" between G-protein-linked receptors. Proc Natl Acad ScI USA 1993; 90: 3103-7.
35. Hebert TE, Moffett S, Morello JP, et al. A peptide derived from a beta2-adrenergic receptor transmembrane domain inhibits both receptor dimerization and activation. Biol Chem 1996; 271: 16384-92.

36. Bai M, Trivedi S, Brown EM. Dimerization of the extracellular calcium-sensing receptor $(\mathrm{CaR})$ on the cell surface of $\mathrm{CaR}$ transfected HEK293 cells. J Biol Chem 1998 ; 273: 23605-10.

37. Cvejic S, Devi LA. Dimerization of the delta opioid receptor: implication for a role in receptor internalization. J Biol Chem 1997; 272 : 26959-64.

38. Romano C, Yang WL, O'Malley KL. Metabotropic glutamate receptor 5 is a disulfide-linked dimer. J Biol Chem 1996 271: 28612-6.

39. Kaupmann K, Malitschek B, Schuler V et al. GABA(B)-receptor subtypes assemble into functional heteromeric complexes. Nature 1998; 396: 683-7.

40. White JH, Wise A, Main MJ, et al. Heterodimerization is required for the formation of a functional GABA(B) receptor. Nature 1998; 396: 679-82

41. Jones KA, Borowsky B, Tamm JA, et al. $G A B A(B)$ receptors function as a heteromeric assembly of the subunits $G A B A(B) R 1$ and GABA(B)R2. Nature 1998; 396: 674-9.

42. Kuner R, Kohr G, Grunewald S, Eisenhardt $G$, Bach A, Kornau HC. Role of heteromer formation in $\mathrm{GABAB}$ receptor function. Science 1999; 283: 74-7.

43. Ng GY, Clark J, Coulombe N, et al Identification of a CABAB receptor subunit $\mathrm{gb} 2$, required for functional $G A B A B$ receptor activity. J Biol Chem 1999; 274 : 7607. 10.

44. Jordan BA, Devi LA. G-protein-coupled receptor heterodimerization modulates receptor function. Nature 1999; 399: 697700.

45. Angers $S$, Salahpour $A$, Joly $E$, et al Detection of beta 2-adrenergic receptor dimerization in living cells using bioluminescence resonance energy transfer (BRET). Proc Natl Acad Sci USA 2000; 97 : 3684-9.

\section{TIRÉS À PART}

M. Bouvier.

\section{ms 2000}

\section{Summary}

Protein scaffoldings

and modular signaling through

seven transmembrane

domains-receptors:

beyond the $\mathrm{G}$ proteins

G protein coupled receptors (GPCR) represent one of the largest families of proteins encoded by the human genome. They mediate cellular communications important in many fundamental physiological processes. Until recently, GPCR function was thought to be mediated almost exclusively via the obligatory activation of hetero-trimeric $\mathrm{G}$ proteins. The development of molecular biological approaches and methods to assess protein-protein interactions have now markedly broadened this view. It is now apparent that proteins such as receptor kinases and arrestins, which quench the signaling function of GPCR, are themselves capable of functioning either as effectors or adaptors that initiate and regulate non-conventional signaling pathways or cell biological processes involved in receptor function. Similarly, the direct interaction of GPCR either through specific sequence motifs, or conformations with small molecular weight $\mathrm{G}$ proteins, enzymes, ion channels or scaffolding proteins indicates a larger than expected diversity in the physiological processes affected by this receptor family. Finally, the realization that homo- and heterodimerization of GPCR as well as interactions with escort proteins can influence their processing, trafficking, ligand binding and signaling specificity points to levels of complexity and diversity that were unsuspected. Unraveling the molecular principles of these interactions and their physiological relevance provides new challenges that will undoubtedly reveal novel targets for the development of therapeutic interventions. 